\title{
Influenza Vaccination Coverage Among Health Care Personnel - United States, 2016-17 Influenza Season
}

\begin{abstract}
Carla L. Black, $\mathrm{PhD}^{1}$; Xin Yue, MPS, MS ${ }^{1}$; Sarah W. Ball, ScD ${ }^{2}$; Rebecca Fink, MPH ${ }^{2}$; Marie A. de Perio, MD 3 ; A. Scott Laney, PhD ${ }^{4}$; Walter W.
\end{abstract} Williams, MD ${ }^{1}$; Megan C. Lindley, MPH' ${ }^{1}$; Samuel B. Graitcer, MD ${ }^{1}$; Peng-Jun Lu, MD, PhD ${ }^{1}$; Rebecca Devlin, MA ${ }^{2}$; Stacie M. Greby, DVM ${ }^{1}$

The Advisory Committee on Immunization Practices (ACIP) recommends that all health care personnel (HCP) receive an annual influenza vaccination to reduce influenza-related morbidity and mortality among HCP and their patients and to reduce absenteeism among HCP (1-4). To estimate influenza vaccination coverage among HCP in the United States during the 2016-17 influenza season, CDC conducted an opt-in Internet panel survey of 2,438 HCP. Overall, $78.6 \%$ of survey respondents reported receiving vaccination during the 2016-17 season, similar to reported coverage in the previous three influenza seasons (5). Vaccination coverage continued to be higher among HCP working in hospitals (92.3\%) and lower among HCP working in ambulatory (76.1\%) and long-term care (LTC) (68.0\%) settings. As in previous seasons, coverage was highest among HCP who were required by their employer to be vaccinated (96.7\%) and lowest among HCP working in settings where vaccination was not required, promoted, or offered on-site (45.8\%). Implementing workplace strategies found to improve vaccination coverage among HCP, including vaccination requirements or active promotion of on-site vaccinations at no cost, can help ensure that HCP and patients are protected against influenza (G).

The Internet panel survey of HCP was conducted for CDC by Abt Associates, Inc. (Cambridge, Massachusetts) during March 28-April 19, 2017, to provide estimates of influenza vaccination coverage during the 2016-17 influenza season. Similar surveys have been conducted since the 2010-11 influenza season, and survey methodology has been described previously (7). HCP were recruited from two preexisting national opt-in Internet sources: Medscape, a medical website managed by WebMD Health Professional Network, ${ }^{*}$ and general population

\footnotetext{
*Physicians, nurse practitioners, physician assistants, nurses, dentists, pharmacists, allied health professionals, technicians, and technologists were recruited from the current membership roster of Medscape. Additional information on Medscape is available at http://www.medscape.com.
}

Internet panels operated by Survey Sampling International (SSI). ${ }^{\dagger}$ Responses were weighted to the distribution of the U.S. population of health care personnel by occupation, age, sex, race/ ethnicity, work setting, and U.S. Census region. ${ }^{\S}$ Because the

\footnotetext{
$\dagger$ Assistants, aides, and nonclinical personnel (e.g., administrators, clerical support workers, janitors, food service workers, and housekeepers) were recruited from general population Internet panels operated by Survey Sampling International. Additional information on Survey Sampling International and its incentives for online survey participants is available at https://www.surveysampling.com.

$\$$ Population control totals of U.S. health care personnel by occupation and work setting were obtained from the Bureau of Labor Statistics, U.S. Department of Labor, Occupational Employment Statistics, May 2015 National IndustrySpecific Occupational Employment and Wage Estimates (https://www.bls.gov/ oes/current/oessrci.htm). Population control totals by other demographic characteristics were obtained from the U.S. Census Bureau, Current Population Survey Monthly Labor Force Data, September 2016 (https://www.bls.gov/cps/ data.htm).
}

\section{INSIDE}

1016 Influenza Vaccination Coverage Among Pregnant Women — United States, 2016-17 Influenza Season

1023 Evaluation of the Impact of Mandating Health Care Providers to Offer Hepatitis C Virus Screening to All Persons Born During 1945-1965 — New York, 2014

1027 Notes from the Field: Outbreak of Campylobacter jejuni Associated with Consuming Undercooked Chicken Liver Mousse - Clark County, Washington, 2016

1028 Announcement

1029 Notice to Readers

1031 QuickStats

Continuing Education examination available at https://www.cdc.gov/mmwr/cme/conted_info.html\#weekly.

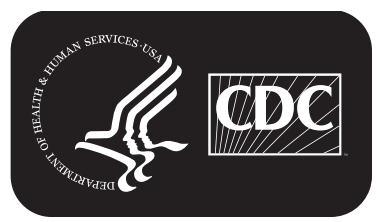

U.S. Department of Health and Human Services Centers for Disease Control and Prevention 
study sample was based on HCP from opt-in Internet panels rather than probability samples, statistical testing was not conducted. 9 An increase or decrease of at least 5 percentage points between seasonal estimates was considered a change; estimates with smaller differences were considered similar.

Among the 2,547 HCP who started the survey from either Medscape or SSI and had eligible responses to the screening questions, 2,493 (97.9\%) completed the survey.** Fifty-four respondents with completed surveys who reported working in "other health care settings" were excluded because examination of their survey responses indicated that they were either unlikely to have contact with patients or to have worked in one of the health care settings of interest for this analysis; in addition, one respondent whose work location was in Canada was excluded. The final analytic sample consisted of 2,438 HCP.

Overall, $78.6 \%$ of respondents reported having received an influenza vaccination during the 2016-17 season. Among all HCP, coverage increased from $63.5 \%$ in the $2010-11$ season to $75.2 \%$ in the $2013-14$ season, and ranged from $77.3 \%$ to $79.0 \%$ in subsequent seasons (Figure) (Table 1). As in previous surveys, coverage in the 2016-17 season was highest

\footnotetext{
Idditional information on obstacles to inference in nonprobability samples is available at http://www.aapor.org/Education-Resources/Reports/Non-ProbabilitySampling.aspx.

** A survey response rate requires specification of the denominator at each stage of sampling. During recruitment of an online opt-in survey sample, such as the Internet panels described in this report, these numbers are not available; therefore, a response rate cannot be calculated. Instead, the survey cooperation rate is provided.
}

among HCP working in hospital settings (92.3\%), followed by HCP working in ambulatory care $(76.1 \%)$, other clinical settings (75.0\%), or LTC (68.0\%) settings. Coverage among HCP working in other clinical settings increased from $69.8 \%$ in $2015-16$ to $75.0 \%$ in $2016-17$; coverage in hospital, ambulatory care, and LTC settings was similar in 2015-16 and 2016-17 (Table 1). Among vaccinated HCP, 76.5\% were vaccinated at their workplace.

Overall, vaccination coverage in 2016-17 was highest among physicians $(95.8 \%)$, nurse practitioners and physician assistants (92.0\%), nurses $(92.6 \%)$, and pharmacists $(93.7 \%)$, and lowest among other clinical HCP (80.0\%), assistants and aides (69.1\%), and nonclinical HCP (73.7\%) (Table 1). However, in hospital settings, vaccination coverage was approximately $90 \%$ or higher in all occupational groups, including assistants and aides and nonclinical personnel.

Overall, $42.3 \%$ of HCP reported a requirement to be vaccinated for the 2016-17 season, an increase over the 2013-14 season but similar to the 2014-15 and 2015-16 seasons. HCP working in hospitals were more likely to report a vaccination requirement $(69.5 \%)$ than were $\mathrm{HCP}$ working in ambulatory care $(39.0 \%)$, LTC (26.2\%), or other clinical settings (22.0\%) (Table 2). HCP working in ambulatory care, LTC, and other clinical settings more often reported that their employer did not require, provide, or promote vaccination $(21.7 \%, 30.5 \%$, and $32.2 \%$, respectively), compared with HCP working in hospital settings $(3.9 \%)$.

The MMWR series of publications is published by the Center for Surveillance, Epidemiology, and Laboratory Services, Centers for Disease Control and Prevention (CDC), U.S. Department of Health and Human Services, Atlanta, GA 30329-4027.

Suggested citation: [Author names; first three, then et al., if more than six.] [Report title]. MMWR Morb Mortal Wkly Rep 2017;66:[inclusive page numbers].

\section{Centers for Disease Control and Prevention Brenda Fitzgerald, MD, Director \\ William R. Mac Kenzie, MD, Acting Associate Director for Science Joanne Cono, MD, ScM, Director, Office of Science Quality \\ Chesley L. Richards, MD, MPH, Deputy Director for Public Health Scientific Services \\ Michael F. Iademarco, MD, MPH, Director, Center for Surveillance, Epidemiology, and Laboratory Services}

\section{MMWR Editorial and Production Staff (Weekly)}

Sonja A. Rasmussen, MD, MS, Editor-in-Chief

Charlotte K. Kent, $\mathrm{PhD}$, MPH, Executive Editor Jacqueline Gindler, MD, Editor

Teresa F. Rutledge, Managing Editor

Douglas W. Weatherwax, Lead Technical Writer-Editor

Soumya Dunworth, PhD, Kristy Gerdes, MPH, Teresa M. Hood, MS, Technical Writer-Editors
Martha F. Boyd, Lead Visual Information Specialist Maureen A. Leahy, Julia C. Martinroe, Stephen R. Spriggs, Tong Yang, Visual Information Specialists

Quang M. Doan, MBA, Phyllis H. King,

Paul D. Maitland, Terraye M. Starr, Moua Yang, Information Technology Specialists
MMWR Editorial Board

Timothy F. Jones, MD, Chairman

Matthew L. Boulton, MD, MPH Virginia A. Caine, MD

Katherine Lyon Daniel, $\mathrm{PhD}$

Jonathan E. Fielding, MD, MPH, MBA

David W. Fleming, MD
William E. Halperin, MD, DrPH, MPH

King K. Holmes, MD, PhD

Robin Ikeda, MD, MPH

Rima F. Khabbaz, MD

Phyllis Meadows, PhD, MSN, RN

Jewel Mullen, MD, MPH, MPA
Jeff Niederdeppe, $\mathrm{PhD}$

Patricia Quinlisk, MD, MPH

Patrick L. Remington, MD, MPH

Carlos Roig, MS, MA

William L. Roper, MD, MPH

William Schaffner, MD 
FIGURE. Percentage of health care personnel (HCP) who reported receiving influenza vaccination, by work setting* and occupation type ${ }^{\dagger}-$ Internet panel surveys, United States, 2010-11 through 2016-17 influenza seasons
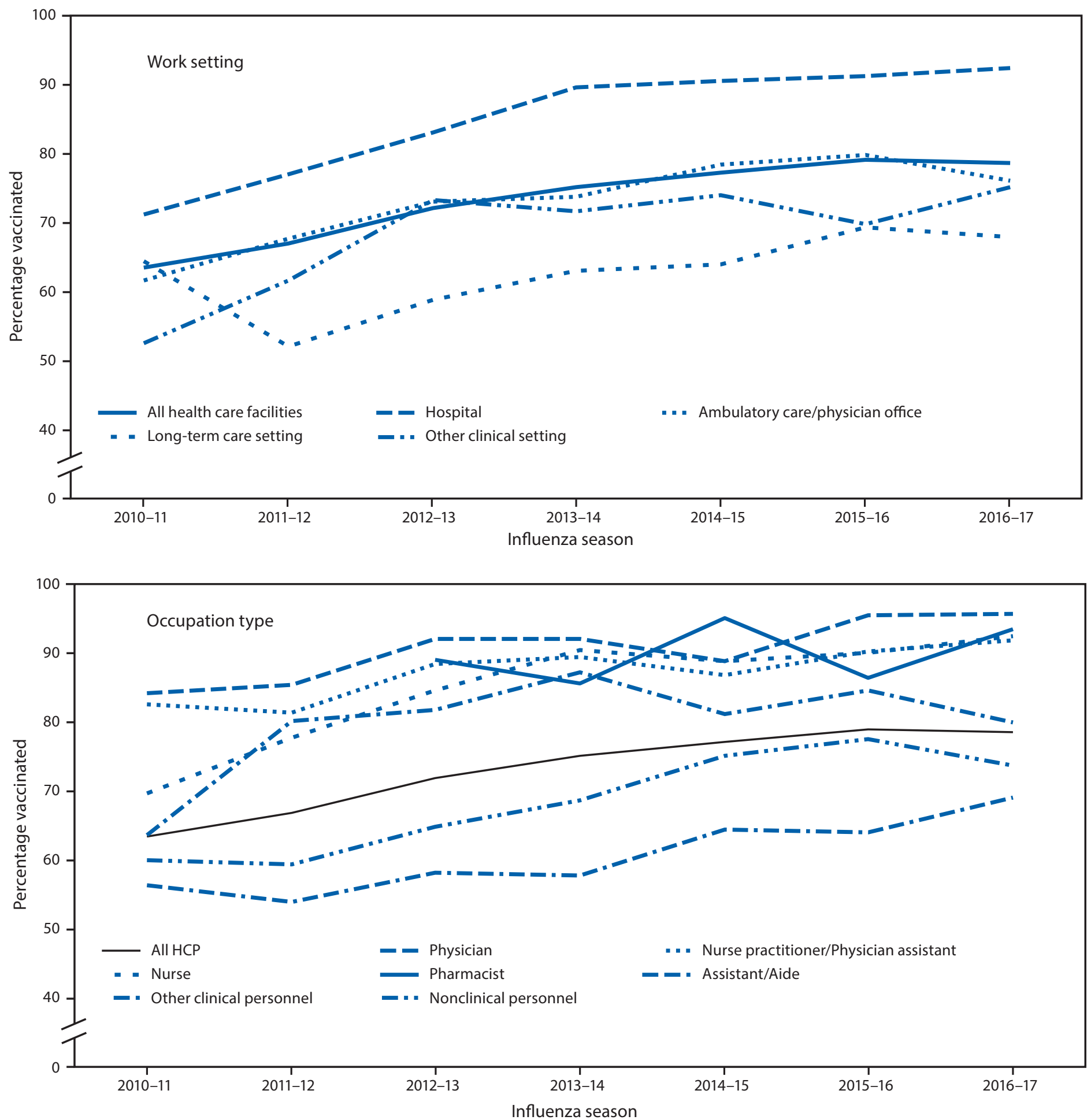

\footnotetext{
* Respondents could select more than one work setting. The "ambulatory care/physician office" category includes physician's office, medical clinic, and other ambulatory care setting. The "other clinical setting" category includes dentist office or dental clinic, pharmacy, laboratory, public health setting, emergency medical services setting, or other setting where clinical care or related services were provided to patients.

† For the 2010-11 season, dentists were included in the physician category. Before the 2012-13 season, separate data on pharmacists were not collected. The "other clinical personnel" category includes allied health professionals, technicians, and technologists. The "nonclinical personnel" category includes administrative support staff members or managers and nonclinical support staff members (e.g., food service workers, laundry workers, janitors, and other members of the housekeeping and maintenance staffs).
} 
TABLE 1. Percentage of health care personnel* (HCP) who reported receiving influenza vaccination, by work setting and occupation type Internet panel surveys, United States, 2015-16 and 2016-17 influenza seasons

\begin{tabular}{|c|c|c|c|c|c|c|c|}
\hline \multirow[b]{2}{*}{ Work setting/Occupation type ${ }^{\dagger}$} & \multicolumn{3}{|c|}{ 2015-16 season } & \multicolumn{3}{|c|}{ 2016-17 season } & \multirow{2}{*}{$\begin{array}{l}\text { Percentage-point } \\
\text { difference } \\
(2015-16 \text { to } \\
2016-17)\end{array}$} \\
\hline & No. in sample & Weighted $\% \S$ & $\begin{array}{l}\text { Weighted } \% \\
\text { vaccinated }\end{array}$ & No. in sample & Weighted \%§ & $\begin{array}{l}\text { Weighted } \% \\
\text { vaccinated }\end{array}$ & \\
\hline Overall & 2,258 & 100 & 79.0 & 2,438 & 100 & 78.6 & -0.4 \\
\hline \multicolumn{8}{|l|}{ Occupational setting, by occupation } \\
\hline Hospital & 803 & 39.7 & 91.2 & 925 & 40.5 & 92.3 & 1.1 \\
\hline Physician & 127 & 3.7 & 99.4 & 129 & 4.2 & 97.8 & -1.6 \\
\hline NP/PA & 50 & 0.9 & 90.0 & 57 & 0.8 & 94.6 & 4.6 \\
\hline Nurse & 95 & 23.8 & 94.6 & 108 & 28.5 & 96.4 & 1.8 \\
\hline Pharmacist & 16 & 0.7 & —? & 121 & 1.2 & 97.4 & - \\
\hline Assistant/Aide & 107 & 8.9 & 88.2 & 118 & 8.4 & 91.1 & 2.9 \\
\hline Other clinical HCP** & 236 & 23.4 & 94.4 & 232 & 22.0 & 90.0 & -4.4 \\
\hline Nonclinical HCP†† & 155 & 38.2 & 87.2 & 144 & 34.6 & 89.7 & 2.5 \\
\hline Ambulatory care/Physician office ${ }^{\S \S}$ & 648 & 27.6 & 79.8 & 718 & 28.8 & 76.1 & -3.7 \\
\hline Physician & 216 & 10.4 & 95.2 & 198 & 9.5 & 94.8 & -0.4 \\
\hline NP/PA & 92 & 2.4 & 89.1 & 110 & 2.7 & 90.0 & 0.9 \\
\hline Nurse & 45 & 20.6 & 88.6 & 48 & 20.5 & 93.3 & 4.7 \\
\hline Pharmacist & 6 & 0.4 & —? & 24 & 0.3 & —? & - \\
\hline Assistant/Aide & 57 & 9.2 & 62.0 & 74 & 9.5 & 74.4 & 12.4 \\
\hline Other clinical HCP** & 135 & 22.0 & 81.7 & 139 & 22.9 & 71.1 & -10.6 \\
\hline Nonclinical HCP†† & 91 & 34.8 & 72.9 & 111 & 34.1 & 63.0 & -9.9 \\
\hline Long-term care setting & 659 & 29.6 & 69.2 & 549 & 29.3 & 68.0 & -1.2 \\
\hline Physician & 17 & 0.8 & -9 & 15 & 0.7 & - & - \\
\hline NP/PA & 7 & 0.2 & — & 7 & 0.2 & - & - \\
\hline Nurse & 23 & 9.6 & - & 22 & 9.7 & - & - \\
\hline Pharmacist & 1 & 0 & - & 6 & 0.1 & - & - \\
\hline Assistant/Aide & 501 & 58.4 & 61.9 & 428 & 59.3 & 66.9 & 5.0 \\
\hline Other clinical $\mathrm{HCP}^{* *}$ & 54 & 7.6 & 85.9 & 26 & 3.8 & - & - \\
\hline Nonclinical HCP ${ }^{\dagger+}$ & 54 & 23.3 & 70.9 & 44 & 26.3 & 60.7 & -10.2 \\
\hline Other clinical setting ๆๆ & 409 & 11.6 & 69.8 & 604 & 12.6 & 75.0 & 5.2 \\
\hline Physician & 4 & 0.6 & —า & 4 & 0.4 & -9 & - \\
\hline NP/PA & 5 & 0.3 & — & 6 & 0.3 & -9 & - \\
\hline Nurse & 15 & 15.2 & - & 15 & 15.3 & - & - \\
\hline Pharmacist & 51 & 9.5 & 85.5 & 243 & 8.7 & 92.4 & 6.9 \\
\hline Assistant/Aide & 42 & 15.4 & 51.2 & 54 & 15.2 & 63.1 & 11.9 \\
\hline Other clinical HCP** & 257 & 32.9 & 72.5 & 240 & 35.3 & 76.5 & 4.0 \\
\hline Nonclinical HCP ${ }^{\dagger+}$ & 22 & 25.3 & -9 & 31 & 24.0 & 69.6 & - \\
\hline \multicolumn{8}{|l|}{ Overall occupation } \\
\hline Physician & 284 & 3.6 & 95.6 & 251 & 3.4 & 95.8 & 0.2 \\
\hline NP/PA & 134 & 1.0 & 90.3 & 154 & 1.0 & 92.0 & 1.7 \\
\hline Nurse & 168 & 18.5 & 90.1 & 167 & 18.6 & 92.6 & 2.5 \\
\hline Pharmacist & 63 & 1.3 & 86.5 & 307 & 1.3 & 93.7 & 7.2 \\
\hline Assistant/Aide & 673 & 23.8 & 64.1 & 641 & 23.9 & 69.1 & 5.0 \\
\hline Other clinical HCP** & 599 & 18.8 & 84.7 & 572 & 18.9 & 80.0 & -4.7 \\
\hline Nonclinical HCP ${ }^{\dagger+}$ & 307 & 32.9 & 77.7 & 315 & 32.6 & 73.7 & -4.0 \\
\hline
\end{tabular}

Abbreviations: NP = nurse practitioner; PA = physician assistant.

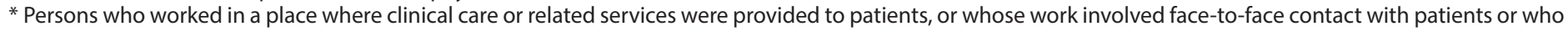
were ever in the same room as patients.

$\dagger$ Respondents could specify working in more than one setting.

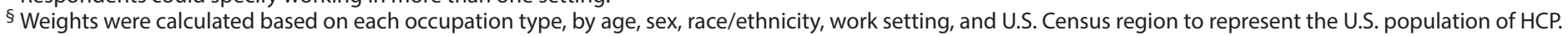

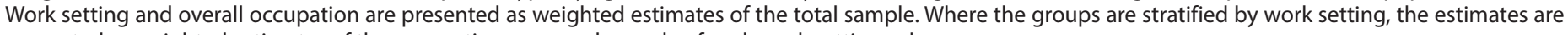
presented as weighted estimates of the occupation group subsample of each work setting subgroup.

I Vaccination coverage estimate not reliable because the sample size was $<30$.

** Allied health professional, technician, or technologist.

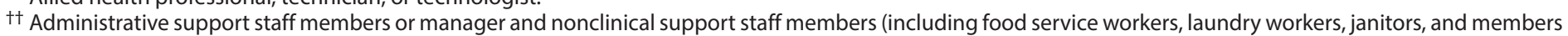
of the housekeeping and maintenance staffs).

$\S \S$ Physician's office, medical clinic, or other ambulatory care setting.

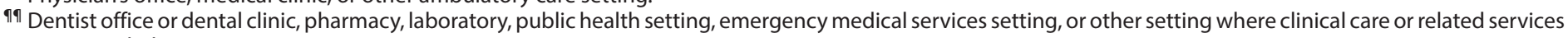
was provided to patients. 
As in previous seasons, vaccination coverage in 2016-17 was highest (96.7\%) among HCP working in settings where vaccination was required, ranging from $90.0 \%$ in LTC settings to $98.3 \%$ in hospital settings (Table 2). Among HCP whose employers did not have a requirement for vaccination, coverage was higher among those who worked in locations where vaccination was available at the worksite at no cost for $>1$ day $(80.3 \%)$ than among those with vaccination available for 1 day only $(73.8 \%)$ or among those who worked in locations where their employer did not provide influenza vaccination on-site at no cost but actively promoted vaccination through other mechanisms $^{\dagger \dagger}(70.4 \%)$. Vaccination coverage was lowest (45.8\%) among HCP working in locations where employers did not require vaccination, provide vaccination on-site at no cost, or promote vaccination (Table 2).

\section{Discussion}

The overall influenza vaccination coverage estimate among HCP was $78.6 \%$ in the $2016-17$ season, an increase of 15 percentage points since the 2010-11 season, but similar to the 2013-14 through 2015-16 seasons (5). As in previous seasons, the highest coverage was among HCP whose workplace had vaccination requirements. In the absence of requirements, HCP with vaccination available at their workplace had higher coverage than those without on-site vaccination. HCP working in hospital settings consistently reported higher vaccination coverage than did those working in other settings and were the most likely to report workplace vaccination requirements and on-site vaccination. Even in occupational groups with lower overall coverage (i.e., assistants, aides, and nonclinical personnel), hospital personnel reported vaccination coverage $\geq 90 \%$. In the 2016-17 season, $93.7 \%$ of HCP working in hospital settings reported either having a vaccination requirement or having on-site vaccination for at least 1 day. Most vaccinated HCP reported being vaccinated at their place of work, underscoring the importance of workplace vaccination availability.

HCP working in LTC settings consistently have lower influenza vaccination coverage than do HCP working in all other health care settings. Influenza vaccination among HCP in LTC settings is especially important because influenza vaccine effectiveness is generally lowest in the elderly, who are at increased risk for severe disease (2). In addition, studies have demonstrated that vaccination of HCP in LTC settings confers a health benefit to patients, including reduced risk for mortality (1-3). In contrast to HCP working in hospitals, only $26.2 \%$ of respondents working in LTC

\footnotetext{
$\dagger^{\dagger \dagger}$ Employer promoted influenza vaccination among employees through public identification of vaccinated persons, financial incentives or rewards to individual persons or groups of employees, competition between units or care areas, free or subsidized cost of vaccination, personal reminders to be vaccinated, or publicizing of the number or percentage of employees receiving vaccination.
}

settings reported having a workplace requirement for vaccination. Among HCP in LTC settings, 30.5\% reported that their employer did not require vaccination, make vaccination available on-site at no cost, or promote vaccination in any way. Workplace vaccination programs that have been successful in increasing coverage in hospital settings could be implemented in LTC and other settings with lower vaccination coverage. Outside of hospital settings, assistants and aides, "other" clinical personnel, and nonclinical HCP have persistently low vaccination coverage. Although some facilities might not prioritize these groups for vaccination programs, especially nonclinical HCP, these personnel often spend considerable time with and in proximity to patients.

The findings in this report are subject to at least three limitations. First, the study used a nonprobability sample of volunteer members of Medscape and SSI Internet panels, which might affect the generalizability of these findings to the U.S. population of HCP. Second, vaccination status and vaccination requirements were self-reported and might be subject to recall bias. Finally, coverage findings from Internet survey panels have differed from population-based estimates from the National Health Interview Survey in past influenza seasons, although trends in coverage were similar across seasons $(8,9)$.

The highest influenza vaccination coverage among HCP continues to be reported in worksites with employer requirements for vaccination. In the absence of vaccination requirements, the findings in this study support the recommendations found in the Guide to Community Preventive Services, which include active promotion of on-site vaccination at no cost or low cost to increase influenza vaccination coverage among HCP (G). Measurement of and feedback about vaccination coverage are additional interventions recommended by the Community Preventive Services Task Force (G). Federal reporting requirements might influence vaccination coverage by occupational setting (10). CDC's National Healthcare Safety Network (NHSN) has included reporting of health care personnel influenza vaccination since 2012. During 2013-2015, the Centers for Medicare \& Medicaid Services (CMS) added requirements to report health care personnel influenza vaccination data through NHSN for acute care hospitals (2013), ambulatory surgery centers (2014), and outpatient hemodialysis facilities (2015), among other settings. ${ }^{\$ \$}$ LTC facilities currently are not covered by CMS quality reporting requirements. LTC employers can use the LTC web-based toolkit 9 developed by $\mathrm{CDC}$ and the National Vaccine Program Office, which provides access to resources, strategies, and educational materials for increasing influenza vaccination among HCP in long-term care settings.

\footnotetext{
$\$ \$$ https://www.cdc.gov/nhsn/cms/index.html.

99 https://www.cdc.gov/flu/toolkit/long-term-care/index.htm.
} 
TABLE 2. Percentage of health care personnel* (HCP) who reported receiving influenza vaccination, by work setting, workplace vaccine availability, and employer vaccine requirements status - Internet panel surveys, United States, 2013-14 through 2016-17 influenza seasons

\begin{tabular}{|c|c|c|c|c|c|c|c|c|c|c|c|c|}
\hline \multirow[b]{2}{*}{ Characteristic } & \multicolumn{3}{|c|}{ 2013-14 season } & \multicolumn{3}{|c|}{ 2014-15 season } & \multicolumn{3}{|c|}{ 2015-16 season } & \multicolumn{3}{|c|}{ 2016-17 season } \\
\hline & $\begin{array}{l}\text { No. in } \\
\text { sample }\end{array}$ & $\begin{array}{c}\text { Weighted } \\
\%^{\dagger}\end{array}$ & $\begin{array}{c}\text { Weighted } \\
\% \text { vaccinated }\end{array}$ & $\begin{array}{l}\text { No. in } \\
\text { sample }\end{array}$ & $\begin{array}{l}\text { Weighted } \\
\%^{\dagger}\end{array}$ & $\begin{array}{c}\text { Weighted } \\
\% \text { vaccinated }\end{array}$ & $\begin{array}{l}\text { No. in } \\
\text { sample }\end{array}$ & $\begin{array}{c}\text { Weighted } \\
\%^{\dagger}\end{array}$ & $\begin{array}{c}\text { Weighted } \\
\% \text { vaccinated }\end{array}$ & $\begin{array}{l}\text { No. in } \\
\text { sample }\end{array}$ & $\begin{array}{c}\text { Weighted } \\
\%^{\dagger}\end{array}$ & $\begin{array}{c}\text { Weighted } \\
\% \text { vaccinated }\end{array}$ \\
\hline $\begin{array}{l}\text { Employer } \\
\text { vaccination } \\
\text { requirement } \$\end{array}$ & 738 & 35.5 & 97.8 & 725 & 40.1 & 96.0 & 841 & 37.8 & 96.5 & 983 & 42.3 & 96.7 \\
\hline Hospital & 520 & 58.2 & 97.7 & 440 & 64.8 & 97.2 & 510 & 61.0 & 96.5 & 644 & 69.5 & 98.3 \\
\hline $\begin{array}{l}\text { Ambulatory care/ } \\
\text { Physician office }\end{array}$ & 252 & 33.6 & 96.4 & 277 & 34.7 & 96.1 & 258 & 33.9 & 98.7 & 305 & 39.0 & 97.2 \\
\hline Long-term care & 88 & 20.1 & 98.4 & 104 & 26.0 & 97.3 & 143 & 23.4 & 93.8 & 142 & 26.2 & 90.0 \\
\hline $\begin{array}{l}\text { Other clinical } \\
\text { setting** }\end{array}$ & 88 & 29.3 & 99.5 & 109 & 35.9 & 85.7 & 101 & 24.9 & 98.5 & 135 & 22.0 & 98.2 \\
\hline $\begin{array}{l}\text { On-site } \\
\text { vaccination } \\
>1 \text { day }^{+\dagger}\end{array}$ & 542 & 25.1 & 80.4 & 407 & 19.1 & 83.9 & 460 & 19.8 & 82.8 & 434 & 15.2 & 80.3 \\
\hline Hospital & 261 & 31.4 & 82.0 & 151 & 21.0 & 86.9 & 173 & 23.8 & 81.8 & 152 & 13.8 & 80.9 \\
\hline $\begin{array}{l}\text { Ambulatory care/ } \\
\text { Physician office }\end{array}$ & 183 & 28.6 & 80.7 & 165 & 23.1 & 87.8 & 152 & 20.8 & 85.1 & 118 & 16.6 & 82.3 \\
\hline Long-term care & 63 & 11.7 & 71.6 & 57 & 12.4 & 67.3 & 96 & 16.1 & 80.4 & 61 & 14.0 & 76.1 \\
\hline $\begin{array}{l}\text { Other clinical } \\
\text { setting }^{* *}\end{array}$ & 107 & 22.0 & 85.0 & 97 & 15.6 & 81.9 & 87 & 12.3 & 84.1 & 155 & 15.6 & 82.8 \\
\hline $\begin{array}{l}\text { On-site } \\
\text { vaccination } \\
1 \text { day }^{\S \S}\end{array}$ & 169 & 7.6 & 61.6 & 230 & 9.8 & 73.6 & 254 & 10.9 & 82.1 & 361 & 14.2 & 73.8 \\
\hline Hospital & 43 & 4.2 & 55.6 & 51 & 7.3 & 72.1 & 70 & 8.3 & 81.1 & 82 & 10.4 & 78.3 \\
\hline $\begin{array}{l}\text { Ambulatory care/ } \\
\text { Physician office }\end{array}$ & 76 & 11.3 & 69.3 & 104 & 10.9 & 80.6 & 76 & 12.8 & 82.9 & 126 & 16.7 & 73.2 \\
\hline Long-term care & 43 & 10.0 & 54.1 & 45 & 10.0 & 67.1 & 77 & 11.5 & 83.0 & 77 & 15.6 & 66.7 \\
\hline $\begin{array}{l}\text { Other clinical } \\
\text { setting }\end{array}$ & 31 & 6.5 & 72.9 & 50 & 10.8 & 80.4 & 54 & 14.2 & 85.2 & 111 & 15.2 & 78.6 \\
\hline $\begin{array}{l}\text { Other vaccination } \\
\text { promotion }{ }^{* * *}\end{array}$ & 226 & 15.5 & 61.9 & 216 & 12.4 & 59.5 & 293 & 13.0 & 67.8 & 206 & 8.2 & 70.4 \\
\hline Hospital & 46 & 5.1 & 80.7 & 24 & 4.4 & —า१ & 39 & 4.6 & 91.0 & 31 & 2.5 & 81.8 \\
\hline $\begin{array}{l}\text { Ambulatory care/ } \\
\text { Physician office }\end{array}$ & 66 & 12.2 & 53.5 & 67 & 10.3 & 60.5 & 62 & 11.9 & 74.0 & 46 & 6.1 & 59.6 \\
\hline Long-term care & 90 & 29.8 & 62.2 & 83 & 21.6 & 58.5 & 139 & 21.4 & 63.4 & 69 & 13.7 & 71.7 \\
\hline $\begin{array}{l}\text { Other clinical } \\
\text { setting }^{* *}\end{array}$ & 50 & 16.9 & 57.5 & 54 & 14.6 & 64.5 & 67 & 16.4 & 54.0 & 77 & 15.1 & 76.7 \\
\hline $\begin{array}{l}\text { No requirement, } \\
\text { on-site } \\
\text { vaccination or } \\
\text { promotion }\end{array}$ & 207 & 16.3 & 36.8 & 336 & 18.7 & 44.0 & 409 & 18.4 & 44.9 & 454 & 20.0 & 45.8 \\
\hline Hospital & 10 & 1.2 & — & 15 & 2.6 & — ११ & 11 & 2.3 & —ภी & 16 & 3.9 & —าๆ \\
\hline $\begin{array}{l}\text { Ambulatory care/ } \\
\text { Physician office }\end{array}$ & 72 & 14.3 & 26.8 & 133 & 21.0 & 46.6 & 100 & 20.6 & 45.0 & 123 & 21.7 & 40.1 \\
\hline Long-term care & 80 & 28.5 & 38.6 & 117 & 30.0 & 36.4 & 204 & 27.7 & 40.6 & 200 & 30.5 & 44.3 \\
\hline $\begin{array}{l}\text { Other clinical } \\
\text { setting }^{* *}\end{array}$ & 51 & 25.3 & 36.9 & 79 & 23.2 & 53.4 & 100 & 32.1 & 43.4 & 126 & 32.2 & 52.8 \\
\hline
\end{tabular}

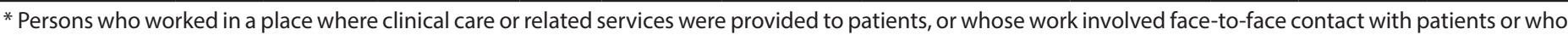
were ever in the same room as patients.

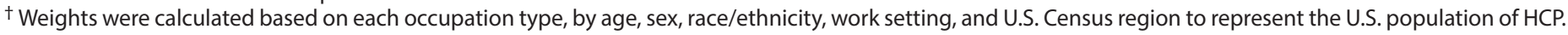

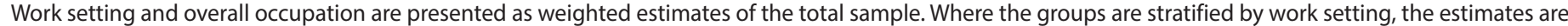
presented as weighted estimates of the occupation group subsample of each work setting subgroup.

$\S$ Includes all respondents who indicated that their employer required them to be vaccinated for influenza.

I Physician's office, medical clinic, or other ambulatory care setting.

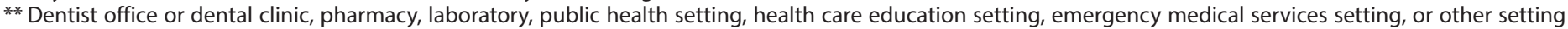
where clinical care or related services was provided to patients.

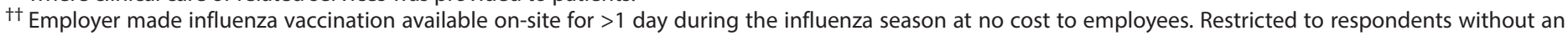
employer requirement for vaccination.

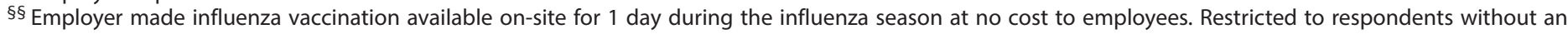
employer requirement for vaccination.

กๆ Vaccination coverage estimate not reliable because the sample size was $<30$.

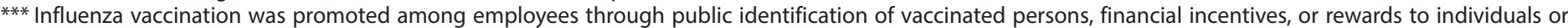

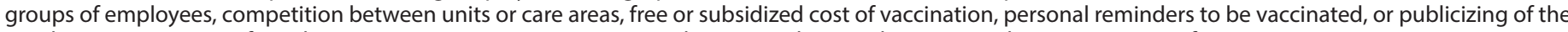

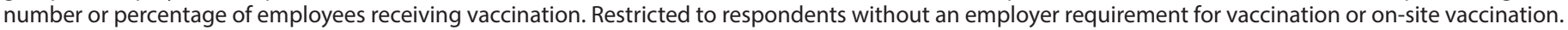




\section{Summary}

What is already known about this topic?

The Advisory Committee on Immunization Practices recommends annual influenza vaccination for all health care personnel (HCP) to reduce influenza-related morbidity and mortality in health care settings. For the 2015-16 influenza season, the estimated overall influenza vaccination coverage among health care personnel was $79.0 \%$.

What is added by this report?

Influenza vaccination coverage among HCP during the 2016-17 influenza season, assessed using an opt-in Internet panel survey, was $78.6 \%$, similar to coverage during the 2015-16 season. Employer vaccination requirements and offering vaccination at the workplace at no cost were associated with higher vaccination coverage. Occupational settings with the lowest influenza vaccination coverage were the least likely to require vaccination or provide vaccination on-site at no cost.

What are the implications for public health practice?

Employer vaccination requirements or, in the absence of requirements, offering influenza vaccination on-site at no cost, can achieve high HCP vaccination coverage. Implementing comprehensive evidence-based worksite intervention strategies is important to ensure HCP and patients are protected against influenza.

\section{Conflict of Interest}

No conflicts of interest were reported.

\footnotetext{
${ }^{1}$ Immunization Services Division, National Center for Immunization and Respiratory Diseases, CDC; ${ }^{2} \mathrm{Abt}$ Associates Inc., Cambridge, Massachusetts; ${ }^{3}$ Division of Surveillance, Hazard Evaluations, and Field Studies, National Institute for Occupational Safety and Health, CDC; ${ }^{4}$ Division of Respiratory Health, National Institute for Occupational Safety and Health, CDC.
}

Corresponding author: Carla L. Black, cblack2@cdc.gov, 404-639-8436.

\section{References}

1. CDC. Immunization of health-care personnel: recommendations of the Advisory Committee on Immunization Practices (ACIP). MMWR Recomm Rep 2011;60(No. RR-7).

2. Hayward AC, Harling R, Wetten S, et al. Effectiveness of an influenza vaccine programme for care home staff to prevent death, morbidity, and health service use among residents: cluster randomised controlled trial. BMJ 2006;333:1241. https://doi.org/10.1136/bmj.39010.581354.55

3. Lemaitre M, Meret T, Rothan-Tondeur M, et al. Effect of influenza vaccination of nursing home staff on mortality of residents: a clusterrandomized trial. J Am Geriatr Soc 2009;57:1580-6. https://doi. org/10.1111/j.1532-5415.2009.02402.x

4. Saxén H, Virtanen M. Randomized, placebo-controlled double blind study on the efficacy of influenza immunization on absenteeism of health care workers. Pediatr Infect Dis J 1999;18:779-83. https://doi. org/10.1097/00006454-199909000-00007

5. Black CL, Yue X, Ball SW, et al. Influenza vaccination coverage among health care personnel-United States, 2015-16 influenza season. MMWR Morb Mortal Wkly Rep 2016;65:1026-31. https://doi. org/10.15585/mmwr.mm6538a2

6. Community Preventive Services Task Force. Vaccination. Atlanta, GA: US Department of Health and Human Services, CDC, Community Preventive Services Task Force; 2008. https://www.thecommunityguide. org/topic/vaccination

7. Black CL, Yue X, Ball SW, et al. Influenza vaccination coverage among health care personnel-United States, 2013-14 influenza season. MMWR Morb Mortal Wkly Rep 2014;63:805-11.

8. CDC. Surveillance of influenza vaccination coverage-United States, 2007-08 through 2011-12 influenza seasons. MMWR Surveill Summ 2013;62(No. SS-04).

9. Williams WW, Lu PJ, O'Halloran A, et al. Surveillance of vaccination coverage among adult populations-United States, 2015. MMWR Surveill Summ 2017;66(No. SS-11). https://doi.org/10.15585/mmwr. ss6611a1

10. Lindley MC, Bridges CB, Strikas RA, et al. Influenza vaccination performance measurement among acute care hospital-based health care personnel-United States, 2013-14 influenza season. MMWR Morb Mortal Wkly Rep 2014;63:812-5. 